PROCEEDINGS OF THE

AMERICAN MATHEMATICAL SOCIETY

Volume 130, Number 6, Pages 1851-1857

S 0002-9939(01)06262-1

Article electronically published on October 23, 2001

\title{
ON FINITENESS OF THE NUMBER OF BOUNDARY SLOPES OF IMMERSED SURFACES IN 3-MANIFOLDS
}

\author{
JOEL HASS, SHICHENG WANG, AND QING ZHOU \\ (Communicated by Ronald A. Fintushel)
}

\begin{abstract}
For any hyperbolic 3-manifold $M$ with totally geodesic boundary, there are finitely many boundary slopes for essential immersed surfaces of a given genus. There is a uniform bound for the number of such boundary slopes if the genus of $\partial M$ is bounded from above.
\end{abstract}

In this paper we consider maps of a compact orientable surface with boundary $(F, \partial F)$ into a compact orientable 3 -manifold with boundary $(M, \partial M)$. An immersion $f:(F, \partial F) \rightarrow(M, \partial M)$ is proper if it takes boundaries to boundaries, so that $f(F) \cap \partial M=f(\partial F)$. A closed curve in a surface is essential if it is not homotopic to a point and a proper arc is essential if it is not homotopic (rel boundary) into $\partial F$. A closed curve is primitive if it is not homotopic to $b^{n}$ where $b$ is a closed curve and $n>1$. A proper immersion of a surface $f(F)$ is essential if no essential closed curve in $F$ is homotopically trivial in $M$, and no essential proper arc in $F$ can be homotoped in $M$ (rel boundary) into $\partial M$. Surfaces which are incompressible and boundary incompressible are essential.

Let $c$ be an essential primitive loop on the boundary $\partial M$ of a compact 3-manifold $M$. If there is a proper immersion of an essential surface $F$ into $M$ such that each component of $\partial F$ is homotopic to a multiple of $c$, we call $c$ a boundary slope of $F$. We do not require $f$ to be an embedding or $c$ to be simple.

We are interested in the following two questions:

Question 1. Given a compact 3-manifold $M$ and a genus $g$, are there finitely many boundary slopes for immersed essential surfaces with genus at most $g$ ?

Question 2. Under what conditions is there a bound for the number of boundary slopes independent of the 3 -manifold?

Results in these directions have been obtained for various classes of 3-manifolds:

(1) If $\partial M$ is a torus and the surfaces are embedded, Hatcher 9 showed that there are only finitely many boundary slopes, without any genus restriction.

(2) When the surfaces are embedded punctured spheres or tori, explicit bounds are known on the number of boundary slopes. These bounds are based on highly

Received by the editors September 2, 1999 and, in revised form, December 28, 2000.

1991 Mathematics Subject Classification. Primary 57N10; Secondary 57M50, 53A10.

Key words and phrases. Boundary slopes, three-dimensional topology, essential surfaces.

The first author was partially supported by NSF grant DMS-9704286.

The second and third authors were partially supported by MSTC and Outstanding Youth Fellowships of NSFC. 
developed combinatorial methods in knot theory and the theory of representations of knot groups. See the survey papers [7], [11] and [16].

(3) When $\partial M$ is a torus and the surfaces are immersed, a positive answer to Question $\square$ has been obtained recently in [ 8$]$. When $M$ is hyperbolic, minimal surface theory is used to derive these bounds. For fixed genus $g$, these bounds are quadratic functions of $g$, independent of $M$. See also recent work of Agol [2].

(4) If $M$ is an irreducible, acylindrical, atoroidal 3-manifold with incompressible boundary and the surfaces are embedded, Scharlemann and $\mathrm{Wu} 15$ gave a positive answer to Question 1 using combinatorial arguments. Recall that a 3-manifold is atoroidal and acylindrical if every properly embedded torus and annulus is boundary parallel.

(5) Suppose $\partial M$ is a torus and the surfaces are immersed. Baker has given examples to show that the bounded genus assumption cannot be dropped [3]. Oertel, using branched surface theory, has found manifolds in which every slope is realized by the boundary of an immersed essential surface [14].

In this note we give a positive answer to Question 1, which extends (3) to the case where $\partial M$ can contain high genus components and generalizes (4) from embedded to immersed surfaces.

Theorem 1. Suppose $M$ is an acylindrical, atoroidal 3-manifold with incompressible boundary. Then for any $g$, there are only finitely many boundary slopes for essential surfaces of genus $g$.

Next we consider the question of obtaining bounds for the number of possible slopes which are independent of the particular manifold we are studying. We define the genus of $\partial M$ to be the sum of the genus of each component of $\partial M$. Only the genus of $\partial M$ is relevant to obtaining a bound on the number of boundary slopes.

Theorem 2. There is a function $n\left(g, g_{\partial}\right)$ such that there are at most $n\left(g, g_{\partial}\right)$ boundary slopes for essential surfaces of genus at most $g$ in an acylindrical, atoroidal 3-manifold with incompressible boundary whose boundary has genus equal to $g_{\partial}$.

Proof of Theorem 1. If $M$ has any 2-sphere boundary components, we can fill them in with balls without changing the number of boundary slopes. Given an embedded 2-sphere, any essential surface can be replaced with one of no greater genus and the same boundary which misses that 2 -sphere, so we can without loss of generality assume that $M$ is irreducible. The number of boundary slopes of essential surfaces lying on a torus boundary component of $M$ is finite by [ 8 , so we restrict our attention to surfaces with boundary on a component of $\partial M$ with genus larger than one. By Thurston's Geometrization Theorem for Haken manifolds, $M$ admits a complete hyperbolic structure of finite volume with totally geodesic boundary [19]. The totally geodesic boundary components consist of the non-torus boundary components of $M$.

Suppose $F$ is an essential immersion having slope $l$. Since $F$ is essential, results of Schoen-Yau, Sacks-Uhlenbeck and Meeks-Yau imply that $F$ can be homotoped to a surface $F^{*}$ whose boundary is the unique geodesic in $\partial M$ homotopic to $\partial F$, and which has least area among all such surfaces [18, [12. Choosing geodesic orthogonal coordinates for the surface $F^{*}$, we have by [6] p. 374] that the metric of $F^{*}$ is given by

$$
d s^{2}=d u^{2}+J^{2}(u, v) d v^{2},(J(u, v)>0 \text { and } J(0, v)=1)
$$


where the $u$-curves (those where $v=$ constant) are geodesics perpendicular to the boundary and the $v$-curves lie on the boundary when $u=0$. The geodesic curvature in $F^{*}$ of a curve $t \rightarrow(u(t), v(t))$ is given by [6, Formula 10.4.7.1],

$$
\frac{1}{\sqrt{E u^{\prime 2}+G v^{\prime 2}}}\left(\frac{d \phi}{d t}+\frac{1}{2 \sqrt{E G}}\left(\frac{\partial G}{\partial u} v^{\prime}-\frac{\partial E}{\partial v} u^{\prime}\right)\right)
$$

where $\phi$ is the angle between the curve and the $u$-curves and the metric on $F^{*}$ is given by $E d u^{2}+G d v^{2}$.

When we consider the $v$-curves, we have $u^{\prime}=0, v^{\prime}=1, \phi=\pi / 2, E=1$ and $G=J^{2}$. Substituting into (2), the geodesic curvature for a $v$-curve $\{u=c\}$ oriented as the boundary of $\{0 \leq u \leq c\}$ is given by $k_{g}=\frac{1}{J} \frac{\partial J}{\partial u}$. Orienting the curve as the boundary of $\{u \geq c\}$ changes the sign and gives

$$
k_{g}=-\frac{1}{J} \frac{\partial J}{\partial u}
$$

The sectional curvature of the surface is [6] Formula 10.5.3.3]:

$$
K=-\frac{1}{J} \frac{\partial^{2} J}{\partial u^{2}} .
$$

A direct computation shows that $k_{g}$ satisfies the equation

$$
\frac{\partial k_{g}}{\partial u}=K+k_{g}^{2} .
$$

Since $M$ is of constant curvature -1 , we have $K=k_{1} k_{2}-1$ by Gauss's Formula [17, p. 179], where $k_{1}$ and $k_{2}$ are the principle curvatures. Since $F$ is a minimal surface, we have $k_{1} k_{2} \leq 0$, and hence $K \leq-1$. Then by (4) it follows that

$$
\frac{\partial^{2} J}{\partial u^{2}} \geq J
$$

Fixing $v=v_{0}$, by (6) we have

$$
\begin{aligned}
& \frac{\partial J}{\partial u}\left(u, v_{0}\right)=\frac{\partial J}{\partial u}\left(u, v_{0}\right)-\frac{\partial J}{\partial u}\left(0, v_{0}\right) \\
= & \int_{0}^{u} \frac{\partial^{2} J}{\partial u^{2}}\left(s, v_{0}\right) d s \geq \int_{0}^{u} J\left(s, v_{0}\right) d s \geq 0 .
\end{aligned}
$$

(11), (3) and (77) imply that $k_{g}<0$ if $u>0$.

Now consider the function $h(u)=-\tanh u$ which is the solution to the differential equation $\frac{d h}{d u}=-1+h^{2}$ with the initial condition $h(0)=0$. Note that $h(u)<0$ when $u>0$ and that the function $k_{g}-h$ satisfies the differential inequality

$$
\frac{d\left(k_{g}-h\right)}{d u}=K+k_{g}^{2}+1-h^{2} \leq k_{g}^{2}-h^{2}=\left(k_{g}-h\right)\left(k_{g}+h\right) .
$$

We want to show that $k_{g}-h \leq 0$. Suppose on the contrary that at some $u \in[0, U]$, $k_{g}-h$ is positive. Pick $u_{0} \in[0, U]$, so that $k_{g}-h$ takes its positive maximum at $u_{0}$. We know $u_{0} \neq 0$ since $\partial F^{*}$ is a geodesic and $k_{g}=h=0$ at 0 . Then $\frac{d\left(k_{g}-h\right)}{d u}$ is zero if $u_{0} \in(0, U)$, and is non-negative if $u_{0}=U$. Hence $\frac{d\left(k_{g}-h\right)}{d u} \geq 0$ at $u_{0}$. Since both $k_{g}$ and $h$ are negative at $u_{0}$, and we assume that $k_{g}-h>0$ at $u_{0}$, we have $\left(k_{g}-h\right)\left(k_{g}+h\right)<0$. This contradicts (8), and so $k_{g} \leq h(u)$ for $u \in[0, U]$.

For $t>0$, let $N_{t}(\partial M)$ be the subset of $M$ with distance $\leq t$ from the boundary. There is a $b>0$ such that $N_{t}(\partial M)$ is a collar of $\partial M$ when $t<b$. Choose $U<b$ in the above and let $N_{U}\left(\partial F^{*}\right)$ be the neighborhood of $\partial F^{*}$ with $u$ coordinates at 
most $U$. Clearly $N_{U}\left(\partial F^{*}\right) \subset N_{b}(\partial M)$. Since $N_{b}(\partial M)$ is a collar of $\partial M$ and the surface $F^{*}$ is essential, $N_{U}\left(\partial F^{*}\right)$ is a collar of $\partial F^{*}$. Letting $F_{U}=\overline{F^{*}-N_{U}\left(\partial F^{*}\right)}$, each component of $\partial F_{U}$ is in the same homotopy class in $M$ as a non-zero multiple of the boundary slope $l$, and $\# \partial F_{U}=\# \partial F^{*}=\# \partial F=n$. By Gauss-Bonnet,

$$
\int_{F_{U}} K d A+\int_{\partial F_{U}} k_{g} d s=2 \pi(\chi(F))=2 \pi(2-2 g-n) .
$$

Let $d$ be the length of the geodesic in the homotopy class of the slope $l$. Then the length of each component of $\partial F_{U}$ is larger than $d$. Since $K \leq-1$ and $k_{g} \leq h<0$ at $U, n h d \geq 2 \pi(2-2 g-n)$. Then we have

$$
d \leq \frac{2 \pi(2 g+n-2)}{-h n} \leq \frac{2 \pi(2 g+1)}{-h} .
$$

Since $g$ is given and $h=h(U)<0$ is a fixed constant, $d$ is bounded above. There are only finitely many homotopy classes of closed curves in $\partial M$ containing elements of length less than a given constant. So for any fixed $g$ there are only finitely many boundary slopes for immersed essential surfaces of genus $g$.

Proof of Theorem 2. We first consider 3-manifolds with totally geodesic boundary of genus at most $g_{\partial}$, and will later consider the possibility of torus boundary components. By Kojima and Miyamoto [10, or by Basmajian [4], there is a lower bound $U^{*}$ to the width of a collar of $\partial M$ for any hyperbolic metric on a manifold $M$ in which $\partial M$ is totally geodesic. Then in the proof of Theorem 1 the boundary has a collar of width $U$ bounded below by $U^{*}$, and $h=h(U) \leq h\left(U^{*}\right)=-\tanh U^{*} \leq 0$. Let $L^{*}=\frac{2 \pi(2 g+1)}{\tanh U^{*}}$. By (9) of Theorem 1 we have that any boundary slope for a surface of genus at most $g$ has length less than $d \leq L^{*}$.

Having now fixed $L^{*}$, we will show that the number of homotopy classes of primitive essential closed curves of length bounded by $L^{*}$ in the $U$-collar of $\partial M$ is uniformly bounded. Since the projection from a collar of the boundary of a hyperbolic manifold with totally geodesic boundary to the boundary is length decreasing, it suffices to consider primitive geodesics on $\partial M$ with length $\leq L^{*}$.

We will use a new constant $\epsilon<L^{*}$ arising from the Collar Lemma ([5], or [13. Theorem 2.18]), and use it to divide the primitive closed geodesics on $\partial M$ into two types:

(1) Those which have length less than $\epsilon$. These will be contained in the thin part of $\partial M$.

(2) Those with length between $\epsilon$ and $L^{*}$. These will be disjoint from the core geodesics in the thin part of $\partial M$.

We will bound the number of each type of geodesic. For $x>0$ set

$$
S(x)=\sinh ^{-1}(1 / \sinh (x / 2)) .
$$

Note that $\lim _{x \rightarrow 0} S(x)=\infty$. For a given simple closed geodesic $c$ with length $l_{c}$ on a hyperbolic surface, let $N(c)=\left\{x: d(x, c) \leq S\left(l_{c}\right)\right\}$. The Collar Lemma states that $N(c)$ is an embedded annular neighborhood of $c$. Moreover if $c_{1}$ and $c_{2}$ are disjoint simple closed geodesics, then $N\left(c_{1}\right)$ and $N\left(c_{2}\right)$ are disjoint. We pick $\epsilon<L^{*}$ so that if $l \leq \epsilon$, then $S(l)>L^{*} / 2$. Then a geodesic of length less than $\epsilon$ has a collar of sufficient width that a curve crossing the collar must have length greater than $L^{*}$. In particular, any two distinct primitive closed geodesics of length $\leq \epsilon$ are disjoint. Let $g$ be the genus of $\partial M$. Then the number of primitive closed geodesics of length 
at most $\epsilon$ is bounded above by $3 g_{\partial}-3$, the number of possible disjoint, non-parallel, essential simple closed curves on a surface of genus $g_{\partial}$.

Decompose the surface $\partial M$ into an $\epsilon$-thin part $\partial M_{(0, \epsilon)}$, which consists of points in $\partial M$ through which there is a non-trivial closed loop of length $\leq \epsilon$ and an $\epsilon$-thick part $\partial M_{[\epsilon, \infty)}$, the points through which every non-trivial loop has length $\geq \epsilon$. While $\partial M_{[\epsilon, \infty)}$ may not be connected, each component has negative Euler characteristic, and so the number of components is bounded by $|\chi(\partial M)|$.

Let $X$ be a component of $\partial M_{[\epsilon, \infty)}$. We now bound the diameter of $X$. The area of $X$ is bounded above by $2 \pi\left(2 g_{\partial}-2\right)$ and its injectivity radius is bounded below by $\epsilon / 2$. We can select a maximal family of points in $X$ having pairwise distance $\geq \epsilon / 2$. Then disks of radius $\epsilon / 2$ around these points cover $X$, and disks of radius $\epsilon / 4$ around them are disjoint and embedded. Let $A(x)$ be the area of the hyperbolic disc with radius $x$. Then the number of such points is at most $N=2 \pi\left(2 g_{\partial}-2\right) / A(\epsilon / 4)$. Form a graph whose vertices are the centers of the $\epsilon / 2$-disks and whose edges join vertices which are at most distance $\epsilon$ apart. This is a connected graph. Between any two points $A$ and $B$ in $X$ we can find a path running from $A$ to the center of an $\epsilon / 2$-disk, then between centers of disks, then to $B$, never reaching a vertex twice. So the diameter $\delta$ of $X$ is at most $N \epsilon+2(\epsilon / 2)=(N+1) \epsilon$.

We can now bound the number of homotopy classes of closed geodesics meeting $X$ with length at most $L^{*}$. Pick a point $x$ in $X$. The lifts of this point are all at least $\epsilon$ from one another. Let $x^{*}$ be a lift of $x$. A closed geodesic in $\partial M$ which intersects $X$ and has length at most $L^{*}$ gives rise to a covering translation that moves $x^{*}$ at most $2 \delta+L^{*}$.

Let $\Gamma_{x}$ be the lattice on the universal covering of $\partial M$ given by the lift of $x$. The number of homotopy classes of geodesics meeting $X$ with length at most $L^{*}$ is bounded by the number of vertices of $\Gamma_{x}$ in the disk of radius $2 \delta+L^{*}$ around $x^{*}$, which is at most

$$
\frac{A\left(2 \delta+L^{*}\right)}{A(\epsilon / 2)}
$$

The number of boundary slopes meeting the thick part of $\partial M$ is bounded by the quantity (10) times the maximal number of components of the thick part, which is $|\chi(\partial M)|=2 g_{\partial}-2$. Adding the number of possible primitive geodesics in $\partial M_{(0, \epsilon)}$ bounds the number of primitive geodesics of length at most $L^{*}$ by

$$
n_{1}\left(g, g_{\partial}\right)=\frac{A\left(2 \delta+L^{*}\right)}{A(\epsilon / 2)}\left(2 g_{\partial}-2\right)+3 g_{\partial}-3 .
$$

If $\partial M$ contains $k$ torus components and the remaining components are totally geodesic with total genus $j$, then $j+k=g_{\partial}$ and there are at most $n_{1}(g, j)+k N(g)$ boundary slopes for proper essential surfaces of genus $g$, where $N(g)$ is the uniform bound for the number of boundary slopes of proper essential surface of genus $g$ on a torus boundary component given in [8, and $n_{1}(g, j)$ is given by (11). We obtain a bound on the number of boundary slopes by considering all possible ways of partitioning $g_{\partial}$ as a sum $j+k$ of non-negative integers, and defining $n\left(g, g_{\partial}\right)$ as the largest number thus obtained.

Remark 3. (1) An explicit value for $\epsilon$ that will satisfy the requirements of Theorem 2 is $\epsilon<\min \left\{L^{*}, 1.75\right\}$. By Lemma 3.1 of [4], we have the lower bound $U^{*}=\frac{1}{4} \log \frac{g_{\partial}+1}{g_{\partial}-1}$. Moreover $A(R)=\frac{4 \pi}{1-\tanh ^{2} R / 2}$. We can combine these to obtain an explicit value for $n\left(g, g_{\partial}\right)$, but this does not appear to give sharp values. 
(2) The assumption of essentiality can be weakened to the assumption that no essential simple closed curve in $F$ is homotopically trivial in $\mathrm{M}$, and no essential properly embedded arc in $F$ can be homotoped (rel its boundary) into $M$. These conditions, which are not known to be equivalent to essentiality, suffice to ensure the existence of a least area map homotopic to $f$.

(3) The least area property is used only to arrange for the normal curvature of the surface to be non-positive. It is possible to use other surfaces that achieve this property, such as Thurston's pleated surfaces.

\section{ACKNOWLEDGMENTS}

These results were obtained while the first two authors were visiting the Mathematical Sciences Research Institute in Berkeley in 1996-97, when the third author visited Peking University in 1998, and when the second author visited the Max Planck Institute in 2000. Research at MSRI was supported in part by NSF grant DMS-9022140. We are indebted to the referee, who made many comments to enhance the paper, including correction of an error in an earlier version of Theorem 2

\section{REFERENCES}

1. C. Adams, Volumes of n-cusped hyperbolic 3-manifolds, J. London Math. Soc. 1988, 38, 2, 555-565. MR 89k:22020

2. I. Agol, Topology of Hyperbolic 3-manifolds, Ph.D. thesis, UCSD, 1998.

3. M. Baker, On the boundary slopes of immersed incompressible surfaces, Ann. Inst Fourier (Grenoble) 46, 1443-1449, (1996). MR 98a:57023

4. A. Basmajian, Tubular neighborhoods of totally geodesic hypersurfaces in hyperbolic manifolds, Invent. Math. 117 (1994), 207-225. MR 95c:57020

5. P. Buser, The collar theorem and examples, Manuscripta Math. 25 (1978), 349-357. MR 80h:53046

6. M. Berger and B. Gostiaux, Differential Geometry: Manifolds, curves and surfaces, GTM 115, Springer Verlag, Berlin, New York, 1988. MR 88h:53001

7. C. Gordon, Dehn surgery on knots, Proceedings of the International Congress of Mathematicians, Vol. I, II (Kyoto, 1990), 631-642, Math. Soc. Japan, Tokyo, 1991. MR 93e:57006

8. J. Hass, H. Rubinstein and S.C.Wang, Immersed surfaces in 3-manifolds, J. Differential Geom. 52 (1999), 303-325. CMP 2000:12

9. A. Hatcher, On the boundary curves of incompressible surfaces, Pacific J. Math. 99 (1982), 373-377. MR 83h:57016

10. S. Kojima and Y. Miyamoto, The smallest hyperbolic 3-manifolds with totally geodesic boundary, J. Differential Geom. 34 (1991), 175-192. MR 92f:57019

11. J. Luecke, Dehn surgery on knots in $S^{3}$, Proc. ICM Vol 2 (Zurich, 1994), 585-594. MR 97h:57019

12. W. Meeks III and S.T. Yau, Topology of three-dimensional manifolds and the embedding problems in minimal surface theory, Ann. of Math. (2) 112 (1980), no. 3, 441-484. MR 83d:53045

13. C. McMullen, Complex dynamics and renormalization, Ann. Math. Studies, No. 135, Princeton University Press, 1994. MR 96b:58097

14. U. Oertel, Boundaries of $\pi_{1}$-injective surfaces, Topology Appl. 78 (1997), 215-234. MR 98f:57029

15. M. Scharlemann and Y. Wu, Hyperbolic manifolds and degenerating handle additions, J. Aust. Math. Soc. (Series A) 55 (1993), 72-89. MR 94e:57019

16. P. Shalen, Representations of 3-manifold groups and its application to topology, Proc. ICM Berkeley, (1986), 607-614. MR 89d:57019

17. M. Spivak, A comprehensive Introduction to Differential Geometry, Vol. 4, Publish or Perish, Inc., Berkeley, 1979. MR 82g:53003d 
18. R. Schoen and S.T. Yau, Existence of incompressible minimal surfaces and the topology of three-dimensional manifolds with nonnegative scalar curvature, Ann. of Math. (2) 110 (1979), 127-142. MR 81k:58029

19. W. Thurston, Three dimensional manifolds, Kleinian groups and hyperbolic geometry, Bull. AMS 6 (1982), 357-388. MR 83h:57019

20. W. Thurston, Geometry and Topology of 3-manifolds, Princeton University Lecture Notes, 1978.

Department of Mathematics, University of California, Davis, California 95616

E-mail address: hass@math.ucdavis.edu

Department of Mathematics, Peking University, Beijing 100871, People's Republic of CHINA

E-mail address: swang@sxx0.math.pku.edu.cn

Department of Mathematics, East China Normal University, Shanghai, 200062, PeoPLE'S REPUBLIC OF CHINA

E-mail address: qzhou@euler.math.ecnu.edu.cn 